\title{
Remark about T-duality of Dp-branes
}

\section{Josef Kluson̆}

Department of Theoretical Physics and Astrophysics, Faculty of Science, Masaryk University, Kotlářská 2, 611 37, Brno, Czech Republic

E-mail: klu@physics.muni.cz

ABSTRACT: This note is devoted to the analysis of T-duality of Dp-brane when we perform T-duality along directions that are transverse to world-volume of Dp-brane.

Keywords: D-branes, String Duality

ArXiv EPrint: 2009.13151 


\section{Contents}

1 Introduction and summary 1

2 Longitudinal T-duality 2

3 Transverse T-duality 4

\section{Introduction and summary}

It is well known that T-duality is central property of string theory, for review, see for example [1]. Generally, if we consider string sigma model in the background with metric $G_{M N}$ and NSNS two form $B_{M N}$ together with dilation $\phi$ and this background possesses an isometry along $d$-directions we find that it is equivalent to string sigma model in T-dual background with dual fields $\tilde{G}_{M N}, \tilde{B}_{M N}$ and $\tilde{\phi}$ that are related to the original fields by famous Buscher's rules $[2,3]$ for generalization to more directions, see for example $[4,5]$.

It is well known that string theories also contain another higher dimensional objects that transform non-trivially under T-duality. In this note we focus on Dp-branes [6, 7], for more recent review, see [10]. Originally Dp-brane was defined with the open string description where the string embedding coordinates obey $p+1$ Neumann boundary conditions and $9-(p+1)-$ Dirrichlet ones at the boundary of the string world-sheet [6]. ${ }^{1}$ It was also shown by Polchinski that Dp-brane transforms into $\mathrm{D}(\mathrm{p}+1)$-brane when T-duality is performed along direction transverse to world-volume of Dp-brane and Dp-brane transforms to $\mathrm{D}(\mathrm{p}$ 1)-brane in case when T-duality is performed along direction that Dp-brane wraps. In other words Dp-brane transforms with very specific way under T-duality transformations.

On the other hand it is remarkable that many aspects of Dp-brane dynamics can be described by its low energy effective action which is famous Dirac-Born-Infeld action [6]. Then one can ask the question whether this description of Dp-brane dynamics could give correct description of T-duality transformation of Dp-brane. This situation is relatively straightforward when we perform T-duality along directions which Dp-brane wraps. This property is known as covariance of Dp-brane action under T-duality transformations as was previously studied in $[8,9]$. We generalize this approach to the T-duality along more longitudinal directions in the next section.

It is important to stress that in order to show full covariance of Dp-brane action with respect to T-duality transformation we should also study how Dp-brane effective action changes when we perform T-duality along transverse directions to its world-volume. The goal of this paper is to perform such an analysis. Our approach is based on previous works

\footnotetext{
${ }^{1}$ We consider Dp-brane in supersymmetric Type IIA or Type IIB theories where the critical dimension of target space time is 10 . Note that $p=0,2,4,6,8$ for Type IIA theory while $p=1,3,5,7,9$ for Type IIB theory.
} 
that consider description of $N$ Dp-branes on the circle [12], for review see [11]. It was shown there that such a configuration should be described by infinite number of Dp-branes on $\mathbf{R}$ which is covering space of $\mathbb{S}^{1}$ when we impose appropriate quotient conditions [11]. Since this description was performed in the context of Matrix theory [13] the low energy effective action describing dynamics of $N$ - Dp-branes was Super Yang-Mills theory (SYM) defined on $p+1$ dimensional world-volume. Then it was shown in [12] that this theory transforms under T-duality into (SYM) defined on $p+2$ dimensional world-volume in T-dual background.

The goal of this paper is to generalize this analysis to the case of full DBI action for Dpbrane in the general background when we study T-duality along transverse directions. It is well known that such a T-duality can be defined when the target space-time fields do not depend on these coordinates explicitly. Since, following previous works, we should consider generalization of DBI action that describes infinite number of Dp-branes in covering space. Such an action is non-abelian generalization of DBI action that was introduced in [14]. Then we follow very nice analysis performed in [15]. Explicitly, we introduce quotient conditions and solve them in the same way as in [15]. We show that non-abelian action for infinite number of Dp-branes transforms to the action for $\mathrm{D}(\mathrm{p}+\mathrm{d})$-brane where $d$ - is number of T-dual directions in the T-dual background where T-dual background fields are related to the original one by generalized Buscher's rules $[4,5]$.

Let us outline our results. We study how Dp-brane transforms under T-duality we consider T-duality either along longitudinal or transverse directions to Dp-brane' worldvolume. We show that in the first case it transforms do $\mathrm{D}(\mathrm{p}-\mathrm{d})$-brane while in the second one it transforms to $\mathrm{D}(\mathrm{p}+\mathrm{d})$-brane when all background fields transform according to generalized Buscher's rules. This fact nicely shows covariance of Dp-brane under T-duality transformations.

Certainly this paper can be extended in many directions. It would be certainly interesting to analyse transverse T-duality transformations in case of the Wess-Zumino term for Dp-brane that determines coupling of Dp-brane to Ramond-Ramond forms. Clearly we should start non-abelian generalization of this term given in [14] when we consider infinite number of Dp-branes on the covering space. This problem is currently under investigation. It would be also nice to analyse non-abelian T-duality on the world-volume of Dp-brane. We hope to return to this problem in future.

This paper is organized as follows. In the next section 2 we introduce Dp-brane action and study T-duality along longitudinal directions. Then in section 3 we consider T-duality along dimensions transverse to Dp-brane world-volume.

\section{Longitudinal T-duality}

In this section we review T-duality transformation of Dp-brane when we perform T-duality along $d$ - longitudinal directions. Explicitly, let us consider DBI action in the general background with the metric $G_{M N}, B_{M N}$ and dilaton $\phi$. This action has the form

$$
S=-T_{p} \int d^{p+1} \xi e^{-\phi} \sqrt{-\operatorname{det}\left(G_{\alpha \beta}+B_{\alpha \beta}+\lambda F_{\alpha \beta}\right)}
$$


where

$$
\lambda=2 \pi \alpha^{\prime}, \quad T_{p}=\frac{2 \pi}{\lambda^{(p+1) / 2}},
$$

where we also defined pull back of $G_{M N}$ and $B_{M N}$ defined as

$$
G_{\alpha \beta}=G_{M N} \partial_{\alpha} x^{M} \partial_{\beta} x^{N}, \quad B_{\alpha \beta}=B_{M N} \partial_{\alpha} x^{M} \partial_{\beta} x^{N},
$$

where $\xi^{\alpha}, \alpha=0,1, \ldots, p$ label world-volume directions of Dp-brane and where $x^{M}, M=$ $0,1, \ldots, 9$ parametrize embedding of DBI action in the target space-time.

Now we would like to perform T-duality along last $d$-directions when we presume that there are directions which Dp-brane wraps. The fact that these directions are longitudinal mean that Dp-brane world-volume coordinates coincide with the target space ones. Explicitly we have

$$
x^{m}=\xi^{m}, \quad m=9-d, \ldots, 9 .
$$

Then we presume that all world-volume fields do not depend on $\xi^{\hat{\alpha}}$ only where $\hat{\alpha}=$ $0,1, \ldots, p-d$. Let us also introduce matrix $E_{M N}=G_{M N}+B_{M N}$. Then we have

$$
E_{\alpha \beta}+\lambda F_{\alpha \beta}=\left(\begin{array}{cc}
E_{\hat{\alpha} \hat{\beta}}+\lambda F_{\hat{\alpha} \hat{\beta}} & E_{\hat{\alpha} n}+\lambda \partial_{\hat{\alpha}} A_{n} \\
E_{m \hat{\beta}}-\lambda \partial_{\hat{\beta}} A_{m} & E_{m n}
\end{array}\right),
$$

where $E_{\hat{\alpha} \hat{\beta}}=E_{\mu \nu} \partial_{\hat{\alpha}} x^{\mu} \partial_{\hat{\beta}} x^{\nu}, \quad \mu, \nu=0,1, \ldots, 9-d$. Then performing standard manipulation with determinant we obtain

$$
\begin{gathered}
\operatorname{det}\left(E_{\alpha \beta}+\lambda F_{\alpha \beta}\right)=\operatorname{det}\left(E_{\hat{\alpha} \hat{\beta}}+\lambda F_{\hat{\alpha} \beta}-\left(E_{\hat{\alpha} m}+\lambda \partial_{\hat{\alpha}} A_{m}\right) \tilde{E}^{m n}\left(E_{n \hat{\beta}}-\lambda \partial_{\hat{\beta}} A_{n}\right)\right) \operatorname{det} E_{m n} \\
=\operatorname{det}\left(E_{\hat{\alpha} \hat{\beta}}-E_{\hat{\alpha} m} \tilde{E}^{m n} E_{n \hat{\beta}}+\lambda F_{\hat{\alpha} \hat{\beta}}+\lambda E_{\hat{\alpha} m} \tilde{E}^{m n} \partial_{\hat{\beta}} A_{n}\right. \\
\left.\quad-\lambda \partial_{\hat{\alpha}} A_{m} \tilde{E}^{m n} E_{n \hat{\beta}}+\lambda^{2} \partial_{\hat{\alpha}} A_{m} \tilde{E}^{m n} \partial_{\hat{\beta}} A_{n}\right) \operatorname{det} E_{m n},
\end{gathered}
$$

and where $\tilde{E}^{m n}$ is inverse to $E_{m n}$ in the sense that $\tilde{E}_{m n} E^{n k}=\delta_{m}^{k}$. As the next step we define $T$-dual coordinates

$$
\tilde{x}_{m} \equiv \lambda A_{m}
$$

Then we can write

$$
\begin{aligned}
E_{\hat{\alpha} \hat{\beta}}-E_{\hat{\alpha} m} \tilde{E}^{m n} E_{n \hat{\beta}}+\partial_{\hat{\alpha}} x_{m} \tilde{E}^{m n} \partial_{\hat{\beta}} x^{n} & =\partial_{\hat{\alpha}} x^{\mu}\left(E_{\mu \nu}-E_{\mu m} \tilde{E}^{m n} E_{n \nu}\right) \partial_{\hat{\beta}} x^{\nu}+\partial_{\hat{\alpha}} \tilde{x}_{m} \tilde{E}^{m n} \partial_{\hat{\beta}} \tilde{x}_{n}, \\
E_{\hat{\alpha} m} \tilde{E}^{m n} \partial_{\hat{\beta}} \tilde{x}_{n} & =\partial_{\hat{\alpha}} x^{\mu} E_{\mu m} \tilde{E}^{m n} \partial_{\hat{\beta}} \tilde{x}_{n}, \\
-\partial_{\hat{\alpha}} \tilde{x}_{m} \tilde{E}^{m n} E_{n \hat{\beta}} & =-\partial_{\hat{\alpha}} \tilde{x}_{m} \tilde{E}^{m n} E_{n \nu} \partial_{\hat{\beta}} x^{\nu},
\end{aligned}
$$

that can be interpreted as an embedding of $\mathrm{D}(\mathrm{p}$-d)-brane in T-dual background with the background fields

$$
\begin{aligned}
\tilde{E}_{\mu \nu} & =E_{\mu \nu}-E_{\mu m} E^{m n} E_{n \nu}, \\
\tilde{E}_{\mu}^{m} & =E_{\mu n} E^{n m}, \quad \tilde{E}_{\nu}^{m}=-E^{m n} E_{n \nu}, \\
e^{-\tilde{\phi}} & =e^{-\phi} \operatorname{det} E_{m n} .
\end{aligned}
$$


Explicitly, the D(p-d)-brane action in T-dual background has the form

$$
S=-T_{p-d} \int_{0}^{\sqrt{\lambda}} d^{d} \xi \int d^{p-d+1} \xi e^{-\tilde{\phi}} \sqrt{-\operatorname{det}\left(\tilde{E}_{\hat{\alpha} \hat{\beta}}+\lambda F_{\hat{\alpha} \hat{\beta}}\right)}
$$

where

$$
\tilde{E}_{\hat{\alpha} \hat{\beta}}=\tilde{E}_{\mu \nu} \partial_{\hat{\alpha}} x^{\mu} \partial_{\hat{\beta}} x^{\nu}+\tilde{E}_{\mu}^{m} \partial_{\hat{\alpha}} x^{\mu} \partial_{\hat{\beta}} \tilde{x}_{m}+\tilde{E}_{\nu}^{m} \partial_{\hat{\alpha}} \tilde{x}_{m} \partial_{\hat{\beta}} \tilde{x}^{\nu}+\partial_{\hat{\alpha}} \tilde{x}_{m} \tilde{E}^{m n} \partial_{\hat{\beta}} \tilde{x}^{n},
$$

and where we defined tension for $\mathrm{D}(\mathrm{p}-\mathrm{d})$-brane in the form

$$
T_{p-d}=T_{p} \int_{0}^{\sqrt{\lambda}} d^{d} \xi=\lambda^{d / 2} T_{p}
$$

Note that the transformation rules for T-dual fields given in (2.9) coincide with the results derived previously in $[4,5]$ and which are now derived independently using covariance of Dp-brane under T-duality transformations.

However in order to see consistency of T-duality covariance of Dp-branes we should also consider opposite situation when we consider Dp-brane in general background and perform T-duality along directions that are transverse to the world-volume of Dp-brane.

\section{Transverse T-duality}

In this section we consider opposite situation when we study Dp-brane in the background that has isometry along $d$-directions in the transverse space to Dp-brane world-volume. The best way how to describe such a Dp-brane in to consider infinite number of Dp-branes on the covering space of torus $T^{d}$ which is $\mathbf{R}^{d}$ and impose appropriate quotient conditions. Further, we should also consider appropriate action for $N$ Dp-branes which is famous Myers non-abelian action [14]

$$
S=-T_{p} \operatorname{Str} \int d^{p+1} \xi e^{-\phi} \sqrt{-\operatorname{det}\left(P\left[E_{\alpha \beta}\right]+P\left[E_{\alpha r} E^{r s}\left(\left(Q^{-1}\right)_{s}^{t}-\delta_{s}^{t}\right) E_{t \beta}\right]+\lambda F_{\alpha \beta}\right) \operatorname{det} Q_{j}^{i}},
$$

where $i, j, k, l, m, n, r, s, t, \ldots=p+1, \ldots, 9$ label directions transverse to the world-volume of $N$ Dp-branes. Note that the location of $N$ - Dp-branes in the transverse space is determined by $N \times N$ Hermitean matrices $\Phi^{m}, m=p+1, \ldots, 9$ and all background fields depend on them so as for example $E_{\alpha \beta}(\Phi)$ and so on. We use convention where $\Phi^{m}$ are Hermitean matrices and field strength $F_{\alpha \beta}$ is defined as

$$
F_{\alpha \beta}=\partial_{\alpha} A_{\beta}-\partial_{\beta} A_{\alpha}+i\left[A_{\alpha}, A_{\beta}\right],
$$

where $A_{\alpha}$ is $N \times N$ Hermitian matrix corresponding to non-abelian gauge field. Finally, $P\left[E_{\alpha \beta}\right]$ is a pull-back of the background $E_{M N}(\Phi)$ defined as

$$
P[E]_{\alpha \beta}=E_{\alpha \beta}+D_{\alpha} \Phi^{r} E_{r \beta}+E_{\alpha r} D_{\beta} \Phi^{r}+D_{\alpha} \Phi^{r} E_{r s} D_{\beta} \Phi^{s},
$$

where $D_{\alpha} \Phi^{m}$ is covariant derivative

$$
D_{\alpha} \Phi^{m}=\partial_{\alpha} \Phi^{m}+i\left[A_{\alpha}, \Phi^{m}\right] .
$$


Note that non-abelian action for $N$ Dp-branes is implicitly defined in the static gauge where world-volume coordinates $\xi^{\alpha}$ coincide with the target space ones $x^{\alpha}$. Finally $\operatorname{Str}$ means symmetrized trace and in order to describe infinite number of Dp-branes we should divide the action (3.1) by the infinite order the quotient group $\mathbf{Z}^{d}$.

Further, $Q^{i}{ }_{j}$ is defined as

$$
Q^{i}{ }_{j}=\delta^{i}{ }_{j}+i \lambda^{-1}\left[\Phi^{i}, \Phi^{k}\right] E_{k j}
$$

and $\left(Q^{-1}\right)^{j}{ }_{k}$ is its inverse in the sense

$$
Q^{i}{ }_{j}\left(Q^{-1}\right)_{k}^{j}=\delta_{k}^{i}
$$

Finally, $P\left[E_{\alpha r} E^{r s}\left(\left(Q^{-1}\right)_{s}^{t}-\delta_{s}^{t}\right) E_{t \beta}\right]$ is defined as

$$
\begin{aligned}
& P\left[E_{\alpha r} E^{r s}\left(\left(Q^{-1}\right)_{s}^{t}-\delta_{s}^{t}\right) E_{t \beta}\right] \\
& =E_{\alpha r} E^{r s}\left(\left(Q^{-1}\right)_{s}^{t}-\delta_{s}^{t}\right) E_{t \beta}+D_{\alpha} \Phi^{m} E_{m r} E^{r s}\left(\left(Q^{-1}\right)_{s}^{t}-\delta_{s}^{t}\right) E_{t \beta} \\
& \quad+E_{\alpha r} E^{r s}\left(\left(Q^{-1}\right)_{s}^{t}-\delta_{s}^{t}\right) E_{t k} D_{\beta} \Phi^{k}+D_{\alpha} \Phi^{m} E_{m r} E^{r s}\left(\left(Q^{-1}\right)_{s}^{t}-\delta_{s}^{t}\right) E_{t n} D_{\beta} \Phi^{n},
\end{aligned}
$$

where $E^{r s}$ is matrix inverse to $E_{m r}$ defined as

$$
E_{m r} E^{r s}=\delta_{m}^{s}
$$

In order to implement T-duality along $d$ transverse directions we follow analysis performed in [15] which we generalize to the case of non-linear non-abelian action (3.1). Let us presume that the background fields do not depend on $x^{A}$ coordinates, where $A=p+1, \ldots, p+d$ and that these coordinates are periodic with period $\sqrt{2 \pi \lambda}$. This is natural if we recognize that all geometrical properties of the background are encoded in the field $E_{M N}$. Then we consider an infinite number of Dp-branes on compact space with coordinates $x^{A}$ when we impose following quotient conditions

$$
\begin{aligned}
& \mathcal{U}_{B}^{-1} \Phi^{A} \mathcal{U}_{B}=\delta_{B}^{A} \sqrt{\lambda}+\Phi^{A} \\
& \mathcal{U}_{B}^{-1} \Phi^{i^{\prime} \mathcal{U}_{B}}=\Phi^{i^{\prime}}, \quad i^{\prime}=p+d+1, \ldots, 9
\end{aligned}
$$

Let us presume that solution of the quotient equation corresponds to operators $\mathcal{U}_{A}$ that commute

$$
\left[\mathcal{U}_{A}, \mathcal{U}_{B}\right]=0
$$

In order to solve (3.9) it is natural to introduce an auxiliary Hilbert space on which $\Phi^{A}$ and $\mathcal{U}_{B}$ act. The simplest way is to introduce Hilbert space of auxiliary functions living on $d$-dimensional torus taking value in $\mathbf{C}^{d}$. Then we take $\mathcal{U}_{A}$ as generators of the functions on $d$-dimensional torus

$$
\mathcal{U}_{A}=e^{i \lambda^{-1 / 2} \sigma_{A}}
$$

where $\sigma_{A}$ are coordinates on the covering space of torus. Then $\Phi^{A}$ has to be equal to

$$
\Phi^{A}=-i \lambda\left(\partial^{A}-i A^{A}\left(\sigma_{A}\right)\right)
$$


since then

$$
\mathcal{U}_{B}^{-1} \Phi^{A} \mathcal{U}_{B}=\lambda^{1 / 2} \delta_{B}^{A}+\Phi^{A}
$$

Using these results we can now proceed to write corresponding action in T-dual background. As the first step we perform following manipulation with the determinant in the action (3.1)

$$
\begin{aligned}
& \operatorname{det}\left(P\left[E_{\alpha \beta}\right]+P\left[E_{\alpha r} E^{r s}\left(\left(Q^{-1}\right)_{s}^{t}-\delta_{s}^{t}\right) E_{t \beta}\right]+\lambda F_{\alpha \beta}\right) \operatorname{det} Q_{j}^{i} \\
& \quad=\operatorname{det}\left(\begin{array}{cc}
P[E]_{\alpha \beta}-P\left[E_{\alpha r} E^{r s} E_{s \beta}\right]+\lambda F_{\alpha \beta} & \mathbf{A}_{\alpha}^{n} \\
\mathbf{B}_{\beta}^{m} & Q^{m n}
\end{array}\right) \operatorname{det} E_{m n},
\end{aligned}
$$

where $Q^{i j}=E^{i j}+i \lambda^{-1}\left[\Phi^{i}, \Phi^{j}\right]$ and where

$$
\mathbf{A}_{\alpha}^{n}=D_{\alpha} \Phi^{k} E_{k r} E^{r n}+E_{\alpha r} E^{r n}, \quad \mathbf{B}_{\beta}^{m}=-E^{m k} E_{k \beta}-D_{\beta} \Phi^{m}
$$

First of all we observe that

$$
P\left[E_{\alpha \beta}-E_{\alpha r} E^{r s} E_{s \beta}\right]=E_{\alpha \beta}-E_{\alpha r} E^{r s} E_{s \beta} .
$$

To proceed further we use the fact that $D_{\beta} \Phi^{A}$ acting on arbitrary function $f(\sigma)$ defined on the space labelled by $\sigma_{A}$ is equal to

$$
D_{\alpha} \Phi^{A} f=\lambda\left(\partial_{\alpha} \Phi^{A}+i\left[A_{\alpha}, \Phi^{A}\right]\right) f=\lambda\left(\partial_{\alpha} A^{A}-\partial^{A} A_{\alpha}\right) f \equiv \lambda F_{\alpha}{ }^{A} f
$$

and hence we can identify $D_{\alpha} \Phi^{A}$ with $\lambda F_{\alpha}{ }^{A}$. Using this identification we obtain

$$
\begin{aligned}
& \mathbf{A}_{\alpha}{ }^{A}=\lambda F_{\alpha}{ }^{A}+E_{\alpha r} E^{r A}, \quad \mathbf{A}_{\alpha}^{i^{\prime}}=D_{\alpha} \Phi^{i^{\prime}}+E_{\alpha r} E^{r i^{\prime}} . \\
& \mathbf{B}_{\beta}^{A}=-E^{A B} E_{B \beta}-E^{A i^{\prime}} E_{i^{\prime} \beta}-\lambda F_{\beta}{ }^{A}, \\
& \mathbf{B}^{i^{\prime}}=-E^{i^{\prime} j^{\prime}} E_{j^{\prime} \beta}-E^{i^{\prime} A} E_{A \beta}-\partial_{\beta} \Phi^{i^{\prime}}
\end{aligned}
$$

and finally

$$
\begin{aligned}
& Q^{A B}=E^{A B}+i \lambda^{-1}\left[\Phi^{A}, \Phi^{B}\right]=E^{A B}+\lambda F^{A B}, \\
& Q^{A i^{\prime}}=E^{A i}+\lambda \partial^{A} \Phi^{i^{\prime}}, \quad Q^{i^{\prime} B}=E^{i^{\prime} B}-\partial^{B} \Phi^{i^{\prime}}, \quad Q^{i^{\prime} j^{\prime}}=E^{i^{\prime} j^{\prime}},
\end{aligned}
$$

where we used (3.12) so that

$$
\left[\Phi^{A}, \Phi^{B}\right]=-i \lambda^{2} F^{A B}, \quad\left[\Phi^{A}, \Phi^{i^{\prime}}\right]=-i \lambda \partial^{A} \Phi^{i^{\prime}} .
$$


Now we return to the first determinant in (3.14) and rewrite it to the form

$$
\begin{aligned}
& \operatorname{det}\left(\begin{array}{cc}
P[E]_{\alpha \beta}-P\left[E_{\alpha r} E^{r s} E_{s \beta}\right]+\lambda F_{\alpha \beta} & \mathbf{A}_{\alpha}^{n} \\
\mathbf{B}_{\beta}^{m} & Q^{m n}
\end{array}\right) \\
& =\operatorname{det}\left(\begin{array}{ccc}
E_{\alpha \beta}-E_{\alpha r} E^{r s} E_{s t}+\lambda F_{\alpha \beta} & \mathbf{A}_{\alpha}^{B} & \mathbf{A}_{\alpha}^{j^{\prime}} \\
\mathbf{B}_{\beta}^{A} & Q^{A B} & Q^{A j^{\prime}} \\
\mathbf{B}^{i^{\prime}} & Q^{i^{\prime} B} & Q^{i^{\prime} j^{\prime}}
\end{array}\right) \\
& =\operatorname{det}\left(\begin{array}{ccc}
E_{\alpha \beta}-E_{\alpha r} E^{r s} E_{s \beta}+\lambda F_{\alpha \beta} & \mathbf{A}_{\alpha}^{B} & \mathbf{A}_{\alpha}^{j^{\prime}} \\
\mathbf{B}_{\beta}^{A}-Q^{A k^{\prime}}\left(Q^{-1}\right)_{k^{\prime} i^{\prime}} \mathbf{B}_{\beta}^{i^{\prime}} & Q^{A B}-Q^{A k^{\prime}}\left(Q^{-1}\right)_{k^{\prime} i^{\prime}} Q^{i^{\prime} B} & 0 \\
\mathbf{B}^{i^{\prime}} & Q^{i^{\prime} B} & Q^{i^{\prime} j^{\prime}}
\end{array}\right) \\
& =\operatorname{det}\left(\begin{array}{ccc}
E_{\alpha \beta}-E_{\alpha r} E^{r s} E_{s \beta}-\mathbf{A}_{\alpha}^{i^{\prime}}\left(Q^{-1}\right)_{i^{\prime} j^{\prime}} \mathbf{B}^{j^{\prime}}{ }_{\beta}+\lambda F_{\alpha \beta} & \mathbf{A}_{\alpha}{ }^{B}-\mathbf{A}_{\alpha}^{j^{\prime}}\left(Q^{-1}\right)_{i^{\prime} j^{\prime}} Q^{j^{\prime} B} & 0 \\
\mathbf{B}_{\beta}^{A}-Q^{A k^{\prime}}\left(Q^{-1}\right)_{k^{\prime} i^{\prime}} \mathbf{B}^{i^{\prime}}{ }_{\beta} & Q^{A B}-Q^{A k^{\prime}}\left(Q^{-1}\right)_{k^{\prime} i^{\prime}} Q^{i^{\prime} B} & 0 \\
\mathbf{B}^{i^{\prime}}{ }_{\beta} & Q^{i^{\prime} B} & Q^{i^{\prime} j^{\prime}}
\end{array}\right) \\
& \equiv \operatorname{det}\left(\begin{array}{ccc}
\mathbf{D}_{\alpha \beta} & \mathbf{D}_{\alpha}^{B} & 0 \\
\mathbf{D}_{\beta}^{A} & \mathbf{D}^{A B} & 0 \\
\mathbf{B}_{B}^{i^{\prime}} & Q^{i^{\prime} B} & Q^{i^{\prime} j^{\prime}}
\end{array}\right)
\end{aligned}
$$

Since $Q^{i^{\prime} j^{\prime}}=E^{i^{\prime} j^{\prime}}$ it is clear that the matrix inverse $\left(Q^{-1}\right)_{i^{\prime} j^{\prime}}$ is equal to $\left(Q^{-1}\right)_{i^{\prime} j^{\prime}}=\tilde{E}_{i^{\prime} j^{\prime}}$ where

$$
\tilde{E}_{i^{\prime} k^{\prime}} E^{k^{\prime} l^{\prime}}=\delta_{i^{\prime}}^{l^{\prime}}
$$

Now we explicitly calculate components of the matrix $\mathbf{D}$ as

$$
\begin{aligned}
\mathbf{D}_{\alpha \beta}= & E_{\alpha \beta}-E_{\alpha r} E^{r s} E_{s \beta}+\partial_{\alpha} \Phi^{i^{\prime}} \tilde{E}_{i^{\prime} j^{\prime}} E^{j^{\prime} r} E_{r \beta}+\partial_{\alpha} \Phi^{i^{\prime}} \tilde{E}_{i^{\prime} j^{\prime}} \partial_{\beta} \Phi^{j^{\prime}} \\
& +E_{\alpha r} E^{r i^{\prime}} \tilde{E}_{i^{\prime} j^{\prime}} E^{j^{\prime} k} E_{k \beta}+E_{\alpha r} E^{r i^{\prime}} \tilde{E}_{i^{\prime} j^{\prime}} \partial_{\beta} \Phi^{j^{\prime}}+\lambda F_{\alpha \beta} \\
= & E_{\alpha \beta}-E_{\alpha A}\left(E^{A B}-E^{A i^{\prime}} \tilde{E}_{i^{\prime} j^{\prime}} E^{j^{\prime} B}\right) E_{B \beta} \\
& +\partial_{\alpha} \Phi^{i^{\prime}} \tilde{E}_{i^{\prime} j^{\prime}} E^{j^{\prime} r} E_{r \beta}+\partial_{\alpha} \Phi^{i^{\prime}} \tilde{E}_{i^{\prime} j^{\prime}} \partial_{\beta} \Phi^{j^{\prime}} \\
& +E_{\alpha r} E^{r i^{\prime}} \tilde{E}_{i^{\prime} j^{\prime}} \partial_{\beta} \Phi^{j^{\prime}}+\lambda F_{\alpha \beta} .
\end{aligned}
$$

To proceed further we observe that

$$
\left(E^{A B}-E^{A i^{\prime}} \tilde{E}_{i^{\prime} j^{\prime}} E^{j^{\prime} B}\right) E_{B C}=\delta_{C}^{A} y
$$

and hence we can identify expression in the bracket with the matrix inverse $\tilde{E}^{A B}$ to $E_{A B}$ so that $\tilde{E}^{A B} E_{B C}=\delta_{C}^{A}$. Further, let us consider following expression

$$
E_{i^{\prime} j^{\prime}}-E_{i^{\prime} A} \tilde{E}^{A B} E_{B j^{\prime}}
$$

and multiply it with $E^{j^{\prime} k^{\prime}}$. Then, after some calculations, we get

$$
\left(E_{i^{\prime} j^{\prime}}-E_{i^{\prime} A} \tilde{E}^{A B} E_{B j^{\prime}}\right) E^{j^{\prime} k^{\prime}}=\delta_{i^{\prime}}^{k^{\prime}}
$$

so that we can identify expression in the bracket with matrix $\tilde{E}_{i^{\prime} j^{\prime}}$

$$
\tilde{E}_{i^{\prime} j^{\prime}}=E_{i^{\prime} j^{\prime}}-E_{i^{\prime} A} \tilde{E}^{A B} E_{B j^{\prime}} .
$$


Using these results we obtain following useful expressions

$$
E^{A i^{\prime}} \tilde{E}_{i^{\prime} j^{\prime}}=-\tilde{E}^{A B} E_{B j^{\prime}}, \quad \tilde{E}_{i^{\prime} j^{\prime}} E^{j^{\prime} B}=-E_{i^{\prime} C} \tilde{E}^{C B}
$$

and

$$
\tilde{E}_{i^{\prime} j^{\prime}} E^{j^{\prime} r} E_{r \beta}=E_{i^{\prime} \beta}-E_{i^{\prime} A} \tilde{E}^{A B} E_{B \beta} .
$$

Using (3.28) and (3.29) we get

$$
\begin{aligned}
\mathbf{D}_{\alpha \beta}= & E_{\alpha \beta}-E_{\alpha A} \tilde{E}^{A B} E_{B \beta}+\partial_{\alpha} \Phi^{i^{\prime}}\left(E_{i^{\prime} \beta}-E_{i^{\prime} A} \tilde{E}^{A B} E_{B \beta}\right) \\
& +\left(E_{\alpha}-E_{\alpha A} \tilde{E}^{A B} E_{B j^{\prime}}\right) \partial_{\beta} \Phi^{j^{\prime}}+\partial_{\alpha} \Phi^{i^{\prime}}\left(E_{i^{\prime} j^{\prime}}-E_{i^{\prime} A} \tilde{E}^{A B} E_{B j^{\prime}}\right) \partial_{\beta} \Phi^{j^{\prime}}
\end{aligned}
$$

and

$$
\begin{aligned}
\mathbf{D}^{A B}= & \tilde{E}^{A B}+\lambda F^{A B}+\partial^{A} \Phi^{i^{\prime}}\left(E_{i^{\prime} j^{\prime}}-E_{i^{\prime} A} \tilde{E}^{A B} E_{B j^{\prime}}\right) \partial^{B} \Phi^{j^{\prime}} \\
& -\tilde{E}^{A C} E_{C j^{\prime}} \partial^{B} \Phi^{j^{\prime}}+\partial^{A} \Phi^{i^{\prime}} E_{i^{\prime} C} \tilde{E}^{C B} .
\end{aligned}
$$

In the same way we obtain

$$
\begin{aligned}
\mathbf{D}_{\alpha}{ }^{B}= & \lambda F_{\alpha}{ }^{B}+E_{\alpha A} \tilde{E}^{A B}+\partial_{\alpha} \Phi^{i^{\prime}} E_{i^{\prime} C} \tilde{E}^{C B} \\
& +\partial_{\alpha} \Phi^{i^{\prime}}\left(E_{i^{\prime} j^{\prime}}-E_{i^{\prime} A} \tilde{E}^{A B} E_{B j^{\prime}}\right) \partial^{B} \Phi^{j^{\prime}}+\left(E_{\alpha i^{\prime}}-E_{\alpha C} \tilde{E}^{C D} E_{D i^{\prime}}\right) \partial^{B} \Phi^{i^{\prime}},
\end{aligned}
$$

and also

$$
\begin{aligned}
\mathbf{D}_{\beta}^{A}= & -\tilde{E}^{A B} E_{B \beta}-\lambda F_{\beta}{ }^{B}-\tilde{E}^{A B} E_{B j^{\prime}} \partial_{\beta} \Phi^{j^{\prime}}+ \\
& +\partial^{A} \Phi^{i^{\prime}}\left(E_{i^{\prime} \beta}-E_{i^{\prime} B} \tilde{E}^{B C} E_{B \beta}\right)+\partial^{A} \Phi^{i^{\prime}} \tilde{E}_{i^{\prime} j^{\prime}} \partial_{\beta} \Phi^{j^{\prime}} .
\end{aligned}
$$

Finally we consider following combinations of determinants that appear under square root in the action $(3.1)$

$$
\begin{aligned}
\operatorname{det} E_{m n} \operatorname{det} E^{i^{\prime} j^{\prime}} & =\operatorname{det}\left(E_{i^{\prime} j^{\prime}}-E_{i^{\prime} A} \tilde{E}^{A B} E_{B j^{\prime}}\right) \operatorname{det} E_{A B} \operatorname{det} E^{i^{\prime} j^{\prime}} \\
& =\operatorname{det} \tilde{E}_{i^{\prime} j^{\prime}} \operatorname{det} E^{i^{\prime} j^{\prime}} \operatorname{det} E_{A B}=\operatorname{det} E_{A B} .
\end{aligned}
$$

Collecting these terms together we obtain final form of $\mathrm{D}(\mathrm{p}+\mathrm{d})$-brane action in T-dual background in the form

$$
S=-\frac{T_{p}}{\lambda^{d / 2}} \int d^{p+1} \xi d^{d} \sigma e^{-\tilde{\phi}} \sqrt{-\operatorname{det} \mathbf{D}},
$$

where we also used the relation between trace over infinite dimensional matrices and integration over coordinates $\sigma$

$$
\operatorname{Tr}=\frac{1}{\lambda^{d / 2}} \int d^{d} \sigma,
$$

and where the matrix $\mathbf{D}$ has following components

$$
\begin{aligned}
\mathbf{D}_{\alpha \beta} & =\tilde{E}_{\alpha \beta}+\partial_{\alpha} \Phi^{i^{\prime}} \tilde{E}_{i \beta}+\tilde{E}_{\alpha j^{\prime}} \partial_{\beta} \Phi^{j^{\prime}}+\partial_{\alpha} \Phi^{i^{\prime}} \tilde{E}_{i^{\prime} j^{\prime}} \partial_{\beta} \Phi^{j^{\prime}}+\lambda F_{\alpha \beta}, \\
\mathbf{D}_{\alpha}^{B} & =\tilde{E}_{\alpha}^{B}+\partial_{\alpha} \Phi^{i^{\prime}} \tilde{E}_{i^{\prime}}{ }^{B}+\tilde{E}_{\alpha j^{\prime}} \partial^{B} \Phi^{j^{\prime}}+\partial_{\alpha} \Phi^{i^{\prime}} \tilde{E}_{i^{\prime} j^{\prime}} \partial^{B} \Phi^{j^{\prime}}+\lambda F_{\alpha}{ }^{B}, \\
\mathbf{D}_{\beta}^{A} & =\tilde{E}_{\beta}^{A}+\tilde{E}_{j^{\prime}}^{A} \partial_{\beta} \Phi^{j^{\prime}}+\partial^{A} \Phi^{i^{\prime}} \tilde{E}_{i^{\prime} \beta}+\partial^{A} \Phi^{i^{\prime}} \tilde{E}_{i^{\prime} j^{\prime}} \partial_{\beta} \Phi^{j^{\prime}}-\lambda F_{\beta}{ }^{A}, \\
\mathbf{D}^{A B} & =\tilde{E}^{A B}+\partial^{A} \Phi^{i^{\prime}} \tilde{E}_{i^{\prime} j^{\prime}} \partial^{B} \Phi^{j^{\prime}}+\tilde{E}_{j^{\prime}}^{A} \partial^{B} \Phi^{j^{\prime}}+\partial^{A} \Phi^{i^{\prime}} \tilde{E}_{i^{\prime}}^{B}+\lambda F^{A B},
\end{aligned}
$$


where we have following components of the background matrix $\tilde{E}$

$$
\begin{array}{llll}
\tilde{E}_{\alpha \beta}=E_{\alpha \beta}-E_{\alpha A} \tilde{E}^{A B} E_{B \beta}, & \tilde{E}_{i^{\prime} \beta}=E_{i^{\prime} \beta}-E_{i^{\prime} A} \tilde{E}^{A B} E_{B \beta}, & \\
\tilde{E}_{\alpha j^{\prime}}=E_{\alpha j^{\prime}}-E_{\alpha A} \tilde{E}^{A B} E_{B j^{\prime}}, & \tilde{E}_{i^{\prime} j^{\prime}}=E_{i^{\prime} j^{\prime}}-E_{i^{\prime} A} \tilde{E}^{A B} E_{B j^{\prime}}, & \\
\tilde{E}_{\alpha}^{B}=E_{\alpha A} \tilde{E}^{A B}, & \tilde{E}_{i^{\prime}}^{B}=E_{i^{\prime} C} \tilde{E}^{C B}, & & \tilde{E}_{i^{\prime} j^{\prime}}=E_{i^{\prime} j^{\prime}}-E_{i^{\prime} A} \tilde{E}^{A B} E_{B j^{\prime}}, \\
\tilde{E}_{\alpha i^{\prime}}=E_{\alpha i^{\prime}}-E_{\alpha C} \tilde{E}^{C D} E_{D i^{\prime}}, & \tilde{E}_{\beta}^{A}=-\tilde{E}^{A B} E_{B \beta}, & \\
\tilde{E}_{i^{\prime} j^{\prime}}=E_{i^{\prime} j^{\prime}}-E_{i^{\prime} A} \tilde{E}^{A B} \tilde{E}_{B j^{\prime}}, & \tilde{E}_{j^{\prime}}^{A}=-\tilde{E}^{A C} E_{C j^{\prime}}, & & \tilde{E}_{i^{\prime}}^{B}=E_{i^{\prime} C} \tilde{E}^{C B},
\end{array}
$$

and where

$$
e^{-\tilde{\phi}}=e^{-\phi} \sqrt{-\operatorname{det} E_{A B}} .
$$

It is important to stress that resulting $\mathrm{D}(\mathrm{p}+\mathrm{d})$-brane propagates in $\mathrm{T}$-dual background where the T-dual background is given by Buscher's rules that are given in equations in (2.11). More explicitly, note that we perform T-duality along directions labelled by $A, B, \ldots$ where $A=p+1, \ldots, p+d$ that should be identify with $m, n, \ldots$ given in the section 2. In the same way $\alpha, \beta=0, \ldots, p$ and $i^{\prime}, j^{\prime}=p+d+1, \ldots, 9$ should be identified with $\mu, \nu$ again given in section 2. Explicitly, if we denote $\mu, \nu, \cdots=\left(\alpha, \beta, i^{\prime}, j^{\prime}, \ldots\right)$ and $A, B, C, \ldots,=m, n, k, \ldots$, then we can rewrite (3.38) into the form

$$
\tilde{E}_{\mu \nu}=E_{\mu \nu}-E_{\mu m} \tilde{E}^{m n} E_{n \nu}, \quad \tilde{E}_{\mu}^{n}=E_{\mu m} \tilde{E}^{m n}, \quad \tilde{E}_{\nu}^{m}=-\tilde{E}^{m n} E_{n \nu}
$$

which exactly match generalized T-duality rules given in (2.11) and hence fully proves covariance of Dp-brane action under T-duality transformations.

\section{Acknowledgments}

This work is supported by the grant "Integrable Deformations" (GA20-04800S) from the Czech Science Foundation (GACR).

Open Access. This article is distributed under the terms of the Creative Commons Attribution License (CC-BY 4.0), which permits any use, distribution and reproduction in any medium, provided the original author(s) and source are credited.

\section{References}

[1] A. Giveon, M. Porrati and E. Rabinovici, Target space duality in string theory, Phys. Rept. 244 (1994) 77 [hep-th/9401139] [INSPIRE].

[2] T.H. Buscher, A Symmetry of the String Background Field Equations, Phys. Lett. B 194 (1987) 59 [INSPIRE].

[3] T.H. Buscher, Path Integral Derivation of Quantum Duality in Nonlinear Sigma Models, Phys. Lett. B 201 (1988) 466 [INSPIRE].

[4] P.H. Ginsparg and F. Quevedo, Strings on curved space-times: Black holes, torsion, and duality, Nucl. Phys. B 385 (1992) 527 [hep-th/9202092] [INSPIRE]. 
[5] F. Quevedo, Abelian and nonAbelian dualities in string backgrounds, in INFN Eloisatron Project: 26th Workshop: From Superstrings to Supergravity, pp. 21-31, 5, 1993 [hep-th/9305055] [INSPIRE].

[6] J. Polchinski, Dirichlet Branes and Ramond-Ramond charges, Phys. Rev. Lett. 75 (1995) 4724 [hep-th/9510017] [INSPIRE].

[7] J. Polchinski, TASI lectures on D-branes, in Theoretical Advanced Study Institute in Elementary Particle Physics (TASI 96): Fields, Strings, and Duality, pp. 293-356, 11, 1996 [hep-th/9611050] [InSPIRE].

[8] K. Kamimura and J. Simon, T duality covariance of superD-branes, Nucl. Phys. B 585 (2000) 219 [hep-th/0003211] [INSPIRE].

[9] J. Simon, T duality and effective D-brane actions, Phys. Rev. D 61 (2000) 047702 [hep-th/9812095] [INSPIRE].

[10] J. Simon, Brane Effective Actions, Kappa-Symmetry and Applications, Living Rev. Rel. 15 (2012) 3 [arXiv:1110.2422] [INSPIRE].

[11] W. Taylor, Lectures on D-branes, gauge theory and M(atrices), in 2nd Trieste Conference on Duality in String Theory, pp. 192-271, 6, 1997 [hep-th/9801182] [INSPIRE].

[12] W. Taylor, D-brane field theory on compact spaces, Phys. Lett. B 394 (1997) 283 [hep-th/9611042] [INSPIRE].

[13] T. Banks, W. Fischler, S.H. Shenker and L. Susskind, $M$ theory as a matrix model: A Conjecture, Phys. Rev. D 55 (1997) 5112 [hep-th/9610043] [INSPIRE].

[14] R.C. Myers, Dielectric branes, JHEP 12 (1999) 022 [hep-th/9910053] [INSPIRE].

[15] D. Brace, B. Morariu and B. Zumino, Dualities of the matrix model from T duality of the Type II string, Nucl. Phys. B 545 (1999) 192 [hep-th/9810099] [INSPIRE]. 